\title{
Effects of Qishenyiqi Dripping Pill on Pulmonary Function in Chronic Obstructive Pulmonary Disease
}

\author{
Lingyun Gao, Hui Ren \\ Sichuan Academy of Medical Sciences \& Sichuan Provincial People's Hospital, Chengdu, P. R. China
}

\author{
Email address: \\ lingyungaosc@163.com (Lingyun Gao)
}

To cite this article:

Lingyun Gao, Hui Ren. Effects of Qishenyiqi Dripping Pill on Pulmonary Function in Chronic Obstructive Pulmonary Disease. American Journal of Internal Medicine. Vol. 4, No. 1, 2016, pp. 1-4. doi: 10.11648/j.ajim.20160401.11

\begin{abstract}
Objective: To investigate the effects of Qishenyiqi Dripping Pill (QDP) on pulmonary function in patients with chronic obstructive pulmonary disease (COPD). Methods: 97 patients with chronic obstructive pulmonary disease admitted to the general wards of Sichuan Academy of Medical Sciences \& Sichuan Provincial People's Hospital randomized plus into experimental group (53 cases) and control group (44 cases) from October 2014 to November 2015. Twice daily QDP 1000mg for experimental group based on the treatment of control group in this study, pulmonary function of two groups was compared. Results: After treatment, FEV1 from $1.36 \pm 0.12 \mathrm{~L}$ to $1.60 \pm 0.22 \mathrm{~L}$ in experimental group, and from $1.42 \pm 0.25$ to $1.51 \pm 0.16 \mathrm{~L}$ in control group. More statistically significant was found in experimental group, $\mathrm{P}$ value $<0.05$. FEV1/FVC from $56.3 \pm 6.39 \%$ to $64.32 \pm 5.21 \%$ in experimental group, and from $56.8 \pm 8.84 \%$ to $57.33 \pm 6.56 \%$ in control group. More statistically significant was found in experimental group, $\mathrm{P}$ value $<0.05$. MMEF from $56.80 \pm 18.22 \%$ to $67.45 \pm 7.55 \%$ in experimental group, and from $55.39 \pm 20.12 \%$ to $60.22 \pm 6.01 \%$ in control group. More statistically significant was found in experimental group, $\mathrm{P}$ value $<0.05$. Conclusions: Qishenyiqi Dripping Pill can significantly improved pulmonary function of patient with chronic obstructive pulmonary disease.
\end{abstract}

Keywords: Qishenyiqi Dripping Pills, Chronic Obstructive Pulmonary Disease, Pulmonary Function

\section{Introduction}

Chronic obstructive pulmonary disease (COPD) is a preventable and treatable disease characterized by progressive and persistent airflow obstruction. COPD exacerbations and the comorbid nature of the disease pose a significant and increasing economic and social burden [1]. Chronic obstructive pulmonary disease (COPD) affects millions of people worldwide and is known to be one of the leading causes of death [2]. Chronic obstructive pulmonary disease is the fourth most common cause of death in the world and will become the third most common in few years [3]. In China, the date of epidemiological investigation in the early 1990s showed that the prevalence rate of COPD over 15years about 3 percent [4], while the prevalence rate rose to $8.2 \%$ in 2005 . How to effectively prevent COPD has become important $t$ issue. Oxidative stress is an important trait in the pathogenesis of chronic obstructive pulmonary disease (COPD). Consequently, targeting oxidative stress is likely to be beneficial as a treatment in COPD. Glutathione (GSH) is an intracellular antioxidant that protects against a variety of different antioxidant species. The increase of lung GSH in COPD is an attempt to counter excess oxidant production but it is inadequate during exacerbations due to the excessive production of ROS. N-acetyl cysteine (NAC) acts as a precursor for the substrate cysteine in synthesis of GSH and also as a mucolytic and anti-inflammatory agent [5]. Increased oxidative stress and inflammation play an important role in the pathogenesis of COPD [6]. In China, QDP, a Chinese medicine formula containing Astragalus membranaceus (Fisch.) Bunge, Salvia miltiorrhiza Bunge, Panax notoginseng (Burkill) can effectively remove oxygen free radicals and antioxidant effects [7]. QDP is a compound Chinese medicine approved by the State Food and Drug Administration of China in 2003 for treatment of cardiac dysfunction [8], which is composed of Astragalus membranaceus (Huangqi), Salvia miltiorrhiza (Danshen), Panax notginseng (Sanqi), and Dalbergiao dorifera (Jiangxiang, DO) [9]. The major active ingredients are astragal side IV (ASIV, from Huanqi), 3, 4-dihydroxy-phenyl lactic acid (DLA, from Danshen), and notoginsenoside R1 (R1, from Sanqi). The primary aim of the present study was to investigate the clinical value of QDP was used to treatment COPD, especially in terms of changes in pulmonary function. 


\section{Subjects and Methods}

\subsection{Study Population and Study Design}

A prospective, two-arm cohort study was conducted in patients with COPD admitted to the general wards of Sichuan Academy of Medical Sciences \& Sichuan Provincial People's Hospital. The study was conducted in accordance with the Declaration of Helsinki and was approved by the ethics committee of Sichuan Academy of Medical Sciences $\&$ Sichuan Provincial People's Hospital. Patients who were diagnosed with COPD according to the published guidelines [10] for COPD and were consecutively admitted to the hospitals from October 2014 to November 2015 were enrolled. The baseline data and demographic information for all the subjects included age, gender, and history of COPD and pulmonary function.

\subsection{Treatment}

Low flow oxygen, expanding the bronchial therapy and antibiotics were given in the control group. Twice daily Qishenyiqi Dripping Pill (Tianjin Tasly Group Co., Ltd. production) $1000 \mathrm{mg}$ for experimental group based on the treatment of control group in this study.

\subsection{Data Collection}

Trained medical personnel visited all recruits at their homes for data collection. All data relating to history of COPD (including age, sex, education level, cognitive function, history of copd and other items) were collected. All information was checked and confirmed by family members who usually lived with the participant.

\subsection{Outcome Measures}

Measures observed in patients with FEV1, FVC, MMEF and other indicators.

\subsection{Statistical Analyses}

Data were presented as mean \pm standard deviation (SD) or number and percentage. All data were computerized for statistical analysis using the Statistical Package for Social Sciences (SPSS) software package, version 11.5 software program. Baseline characteristics were analyzed using Chi Square $\left(\mathrm{X}^{2}\right)$-test or Fisher's exact test; All tests were two tailed and a $\mathrm{P}$-value $\leq 0.05$ was considered statistically significant.

\section{Results}

From October 2014 to November 2015, a total of 97 patients with COPD were ultimately enrolled in this study. all cases were in line edition 2014 "Chronic Obstructive Pulmonary Disease Global Initiative" diagnostic criteria [10], the patients randomized into experimental group (53 cases, 28 males and 25 females) and control group (44 cases, 24 males and 20 females). The baseline characteristics of the patients are shown in Table 1. No statistically significant differences was found in age, gender, history of COPD, and other aspects of pulmonary function in two groups, $\mathrm{P}$ value $>0.05$.

Table 1. Baseline data of patients with copd in two groups.

\begin{tabular}{|c|c|c|c|c|c|}
\hline Baseline & experimental group & control group & $\mathbf{T} / \mathbf{t}^{\prime}$ value & Value & $95 \%$ CI \\
\hline $\mathrm{N}$ & 53 & 44 & & & \\
\hline Gender (male / female) & $28 / 25$ & $24 / 20$ & & & \\
\hline Age (years) & $69.22 \pm 12.35$ & $68.88 \pm 14.56$ & -0.36 & 0.953 & $-14.23,12.96$ \\
\hline History of COPD (years) & $13.36 \pm 7.26$ & $16.99 \pm 8.23$ & 0.767 & 0.432 & $-7.33,15.98$ \\
\hline FEV1 (L) & $1.36 \pm 0.12$ & $1.42 \pm 0.25$ & 0.95 & $>0.05$ & $0.89,1.55$ \\
\hline FEV1/FVC (\%) & $56.3 \pm 6.39$ & $56.8 \pm 8.84$ & 0.43 & $>0.05$ & $-3.56,7.59$ \\
\hline MMEF (\%) & $56.80 \pm 18.22$ & $55.39 \pm 20.12$ & 0.55 & $>0.05$ & $-20.31,17.88$ \\
\hline
\end{tabular}

Table 2. Pulmonary function after treatment in two groups.

\begin{tabular}{lllll}
\hline group & N & FEV1(L) & FEV1/FVC (\%) & MMEF (\%) \\
\hline experimental group & 53 & $1.60 \pm 0.22$ & $64.32 \pm 5.21$ & $67.45 \pm 7.55$ \\
control group & 44 & $1.51 \pm 0.16$ & $57.33 \pm 6.56$ & $60.22 \pm 6.01$ \\
P value & & $<0.05$ & $<0.05$ & $<0.05$ \\
\hline
\end{tabular}

After treatment, FEV1 from $1.36 \pm 0.12 \mathrm{~L}$ to $1.60 \pm 0.22 \mathrm{~L}$ in experimental group, and from $1.42 \pm 0.25$ to $1.51 \pm 0.16 \mathrm{~L}$ in control group. More statistically significant was found in experimental group, $\mathrm{P}$ value $<0.05$. FEV1/FVC from $56.3 \pm 6.39 \%$ to $64.32 \pm 5.21 \%$ in experimental group, and from $56.8 \pm 8.84 \%$ to $57.33 \pm 6.56 \%$ in control group. More statistically significant was found in experimental group, $\mathrm{P}$ value $<0.05$. MMEF from $56.80 \pm 18.22 \%$ to $67.45 \pm 7.55 \%$ in experimental group, and from $55.39 \pm 20.12 \%$ to $60.22 \pm 6.01 \%$ in control group. More statistically significant was found in experimental group, $\mathrm{P}$ value $<0.05$ (see table 2).

\section{Discussion}

COPD is the fourth commonest cause of death in the world and soon will be the third one in a few years. Acute exacerbation is a frequent event in the natural course of COPD, and is characterized by acute aggravations of respiratory symptoms such as dyspnea, cough with or without sputum which require specific regimens [1]. One of the earliest and most quoted definitions is that of Anthonisen [11], which is based on an increase in symptoms of dyspnoea, sputum volume and sputum purulence with or without symptoms of upper respiratory infection and then subdivided depending on the number of symptoms present. This definition is based upon the infective exacerbation of the COPD patients. These episodes vary in severity from mild exacerbations (normally managed at home by the patient) to moderate exacerbations 
(requiring consultation with primary care physicians) and severe exacerbations (needing hospitalization).

Oxidative stress is an important trait in the pathogenesis of COPD. Consequently, targeting oxidative stress is likely to be beneficial as a treatment in COPD. Increased oxidative stress and inflammation play an important role in the pathogenesis of COPD [6]. The drugs of anti-oxidation activity may be effective in treating COPD; Nanshan Zhong found that in Chinese patients with moderate-to-severe COPD, long-term use of $\mathrm{N}$-acetyl cysteine $600 \mathrm{mg}$ twice daily can prevent exacerbations, especially in disease of moderate severity [12]. Liu analysis ten RCT studies about anti-oxidation treatment, all the study involving over 3000 patients were identified. Compared with placebo, the use of mucolytic and antioxidant agents was associated with a reduction of 0.36 exacerbations per patient per year $(95 \%$ CI 0.47 to 0.26$)$. The subgroup analysis restricting to studies without concomitant ICS indicated that there was a larger decrease between the two groups (WMD $=-0.58,95 \% \mathrm{CI}-0.89--0.27$ ). The analysis of NAC dose on the effect indicated that high-dose NAC treatment could significantly reduce the exacerbations (WMD $=-0.39,95 \% \mathrm{CI}-0.61--0.16, \mathrm{z}=3.33, \mathrm{P}=0.001$ ). While in the low-dose NAC group, only those studies with low rate of concomitant ICS showed the effects , $\mathrm{P}=0.001$, the difference was not significant in studies with high rate of concomitant ICS, $\mathrm{P}=0.617$. There was no difference in FEV1 improvement, $\mathrm{P}=0.626$, but $\mathrm{FEF} 25 \%-75 \%$ showed a significant improvement, $\mathrm{P}=0.004$, so the conclusion of the study was the use of mucolytic and antioxidant agents is associated with a reduction in the number of exacerbations. The effects may be larger in those patients without concomitant ICS. High-dose NAC treatment may be more effective than low-dose group, while the latter may be effective only when the rate of concomitant ICS was low [13].

In this prospective study, FEV1, FEV1/FVC and MMEF have improved in two groups after treatment. But more significantly was found in the experimental group, $\mathrm{P}$ value $<0.05$. The result was similar to NAC study. We found that QDP are associated with better clinical outcomes in those patients admitted to the general ward with a physician diagnosis of copd.

QDP formula containing Astragalus membranaceus (Fisch.) Bunge, Salvia miltiorrhiza Bunge, Panax notoginseng (Burkill) can effectively remove oxygen free radicals and antioxidant effects [7]. An increasing number of studies have focused on the relationship between QDP and coronary heart disease. Chen found that pretreatment of QDP in Spraguedawley rats improves ventricular function and energy metabolism and reduces oxidative stress via ameliorating multiple mitochondrial dysfunctions during I/R injury [14], Huang found that readmission rates of coronary heart disease was reduced after QDP treatment [15]. Another study has focused on the relationship between QDP and chronic pulmonary heart disease. LV Libo found that Blood gas analysis and blood coagulation index ( $\mathrm{SpO} 2, \mathrm{Fg}, \mathrm{DD})$ level changes in the treatment group and control group were improved $(\mathrm{P}<0.05)$, the treatment group by QDP is better than that of control group
$(\mathrm{P}<0.05)$. Pulmonary function index pulmonary function index in the two groups were improved $(\mathrm{P}<0.01)$, the treatment group and control group improved with equivalence $(\mathrm{P}>0.05)$ [16]. There are also studies focus on QDP and idiopathic pulmonary fibrosis. Long tao found that there was significant difference in clinical effect $(\mathrm{P}<0.05)$ after QDP treatment between the treatment group and control group, and there were significant differences in $\mathrm{FVC} \%$, FEV1\%, DLCO $\%$ and $\mathrm{PaO} 2$ between the two groups $(\mathrm{P}>0.05)$. QDP is a significant effect for idiopathic pulmonary fibrosis [17]. However, there has no study about copd treatment by QDP. In this prospective study, we found that pulmonary function was improved in those patients admitted to the general ward with a physician diagnosis of copd after QDP treatment. Significantly different in pulmonary function was found in our study, long-term use QDP may improve the prognosis of patients with COPD. FEV1 in experimental group was $1.60 \pm 0.22 \mathrm{~L}$ after QDP treatment, It was higher than control group $(1.51 \pm 0.16 \mathrm{~L}), \quad \mathrm{P}$ value $<0.05$. FEV1/FVC in experimental group was $64.32 \pm 5.21 \%$ after QDP treatment, It was higher than control group $(57.33 \pm 6.56 \%), \mathrm{P}$ value $<0.05$. MMEF in experimental group was $67.45 \pm 7.55 \%$ after QDP treatment, It was higher than control group $(60.22 \pm 6.01 \%), \mathrm{P}$ value $<0.05$. It means that $\mathrm{QDP}$ can improve pulmonary function and alleviate the condition of patients with COPD.

This observation indicates that QDP can improve pulmonary function of COPD. This study is the first study about QDP treatment COPD, future studies are needed to expand the sample size to confirm its influence in the prognosis of patients with COPD.

\section{Conclusion}

In summary, Qishenyiqi Dripping Pill can significantly improved pulmonary function of patient with chronic obstructive pulmonary disease.

\section{Acknowledgements}

The authors thank the staff of the Department of Respiratory Medicine, Sichuan Academy of Medical Sciences, Sichuan Provincial People's Hospital.

\section{References}

[1] Global Strategy for the Diagnosis, Management and Prevention of COPD, Global Initiative for Chronic Obstructive Lung Disease (GOLD). (Updated 2015) [EB/OL]. http://www.goldcopd.org/ Global Initiative for Chronic Obstructive Lung Disease.

[2] Bose P, Bathri R, Kumar L, et al. Role of oxidative stress \& transient receptor potential in chronic obstructive pulmonary disease. Indian J Med Res. 2015; 142(3): 245-60.

[3] Lingyun Gao, Hui Ren, et al. IGC in the treatment of hyperglycemia in patients with an acute exacerbation of COPD: a prospective study. Journal of Aging Research and Clinical Practice. 2014, 3(3) 159-162. 
[4] Dongjie Ding, quanying He. Advances in diagnosis and treatment of COPD. Chinese journal of tuberculosis and respiratory disease. $1995,18: 227-281$.

[5] Matera MG, Calzetta L, Cazzola M. Oxidation pathway and exacerbations in COPD: the role of NAC. Expert Rev Respir Med. 2015; 10(1): 89-97.

[6] Lingyun Gao, et al. Advances in oxidative stress and chronic obstructive pulmonary disease, Chinese medical record, 2010, 11(2): 23-27.

[7] Wang J, Lu L, Wang Y, et al. Qishenyiqi Dripping Pill attenuates myocardial fibrosis in rats by inhibiting RAAS-mediated arachidonic acid inflammation. J Ethnopharmacol. 2015, 24; 176: 375-84.

[8] Wang Y. et al. Development of fluorescence imaging-based assay for screening cardio protective compounds from medicinal plants. Anal. Chim. Acta. 2011, 702, 87-94.

[9] Zhang Y., Shi P., Yao H., et al. Metabolite profiling and pharmacokinetics of herbal compounds following oral administration of a cardiovascular multi-herb medicine (Qishenyiqi pills) in rats. Curr. Drug Metab. 2012, 13, 510523.

[10] Global strategy for the diagnosis, management, and prevention of chronic obstructive pulmonary disease (updated 2014) [EB/OL]. http://www.goldcopd.org/Global Initiative for Chronic Obstructive Lung Disease.

[11] Anthonisen NR, Manfreda J, Warren CP, et al. Antibiotic therapy in exacerbation of COPD. Ann Intern Med. 1987; 166: 196-204.
[12] Jin-Ping Zheng, Fu-Qiang Wen, Nan-Shan Zhong, et al. Twice daily $\mathrm{N}$-acetyl cysteine $600 \mathrm{mg}$ for exacerbations of chronic obstructive pulmonary disease (PANTHEON): a randomized, double-blind placebo-controlled trial. Lancet Respire Med 2014; 2: 187-94.

[13] Li X, Sun H, Liu C, Kang J. Mucolytic and antioxidant agents for exacerbations of chronic obstructive pulmonary disease: A meta-analysis. Zhonghua Jie He He Hu Xi Za Zhi. 2015 Aug; 38(8): $600-6$.

[14] Chen JR, Wei J, Wang LY, et al. Cardio protection against ischemia/reperfusion injury by Qi Shen Yi Qi Pill $\mathbb{R}$ viaameliorate of multiple mitochondrial dysfunctions. Drug Des Devel Ther. 2015 Jun 15; 9: 3051-66.

[15] Hui Huang, li hua Li, et al. study of Qishenyiqi Dripping Pill treatment of chronic pulmonary heart disease. Journal of integrative medicine heart cerebrovascular disease. 2008, 6(5): 509-510.

[16] LV li bo. Qishenyiqi Pill Add with Enoxaparin in Patients w ith Chronic O bstructive Pulm onary Disease Study of Prothrom botic State R andom Parallel C ontrol. Journal of practical tranitional Chinese internal medicine. 2013, 27(9): 29-31.

[17] Longtao, li ying. Effects of Qishenyiqi Dripping Pills on Idiopathic Pulmonary Fibrosis. Chinese general practice, 2009, 12(3): 430-432. 